\title{
Fluorine-18 fluorodeoxyglucose PET/CT is a suitable instrument to show the effects of lipid metabolism disorders on metabolic networks in the living organism
}

\author{
Oana C. Kulterer, MD, a and Marcus Hacker, $M D^{\mathrm{a}}$ \\ a Division of Nuclear Medicine, Department of Biomedical Imaging and Image-Guided Therapy, \\ Medical University Vienna, Vienna, Austria
}

Received Jan 12, 2021; accepted Jan 12, 2021

doi: $10.1007 / \mathrm{s} 12350-021-02543-8$

See related article, pp. 1405-1414

In this issue of the Journal of Nuclear Cardiology, Toutouzas $\mathrm{MD}, \mathrm{PhD}$ and colleagues in their study "Visceral adipose tissue phenotype and hypoadiponectinemia are associated with aortic Fluorine-18 fluorodeoxyglucose uptake in patients with familial dyslipidemias" add new data to the still emerging field of adipose tissue phenotype and hypoadiponectinemia and their associations with arterial inflammation in patients with familial combined hyperlipidemia $(\mathrm{FCH})$ or heterozygous familial hyperlipidemia (heFH). To enlighten the complex interaction between fat deposition and inflammation of involved tissues, Toutouzas and coworkers made use of the possibilities of Fluorine-18 fluorodeoxyglucose (FDG) PET/CT by quantifying not only the FDG uptake from PET, but also the volumes of abdominal visceral (VAT) and subcutaneous adipose tissue (SAT).

Familial dyslipidemias are a group of diseases characterized by hypercholesterolemia, hypertriglyceridemia, or a combination of both accompanied by abnormal high-density lipoprotein cholesterol levels. Familial hypercholesterolemia (FH) is one the most common inherited metabolic disorders and it is

Reprint requests: Marcus Hacker, MD, Division of Nuclear Medicine, Department of Biomedical Imaging and Image-Guided Therapy, Medical University Vienna, Währinger Gürtel 18-20, 1090 Vienna, Austria; Marcus.hacker@meduniwien.ac.at

J Nucl Cardiol 2022;29:1415-8.

$1071-3581 / \$ 34.00$

Copyright (c) 2021 American Society of Nuclear Cardiology. characterized by elevated low-density lipoprotein cholesterol (LDL-C) from birth leading to early onset of cardiovascular disease. ${ }^{1}$ It is an autosomal dominant disorder with a gene dosage effect. Homozygous patients have higher LDL-C levels and an early onset of cardiovascular disease as compared to heterozygous patients. The prevalence of homozygous $\mathrm{FH}$ is about $1: 250,000$ whereas the prevalence of heterozygous is about 1:250. In these patients, mutations in four different genes can occur: LDL receptor, which is the most common, the LDL receptor binding region of apoliporotein B (Apo B), a rare gain of function of proprotein convertase subtilin/kexin 9 (PCSK9), and loss-of-function mutations in the LDL receptor adaptor protein (LDLRAP). ${ }^{1}$ In comparison to familial hypercholesterolemia, the familial combined hyperlipidemia $(\mathrm{FCH})$ is the most common inherited form of dyslipidemia with a prevalence about 1:100. FCH is accompanied by a multitude of metabolic defects such as adipose tissue dysfunction, hepatic steatosis, insulin resistance, and impaired metabolism of lipoprotein particles. ${ }^{2}$

\section{WHITE ADIPOSE TISSUE}

White adipose tissue is a complex organ, with a multitude of functions, the most important being its energy storage function and its cytokine secretory function. White adipose tissue secretes cytokines like leptin, an adipokine which is secreted during a positive energy balance in order to reduce food intake and increase energy expenditure, proinflammatory (interleukin-6), or anti-inflammatory (adiponectin, resistin) cytokines. ${ }^{3}$ Obesity occurs as an imbalance between energy intake and energy expenditure and is accompanied by an increase in body fat and altered secretory function as well as adipose tissue fibrosis and hypoxia. 
White adipose tissue dysfunction is associated with fat disposition in organs that play pivotal roles in glucose homeostasis, like the liver, skeletal muscle, and heart, which in turn leads to systemic insulin resistance and increased risk of type 2 diabetes. ${ }^{4}$ Adipose tissue dysfunction is thus associated with altered secretory function leading to impaired secretion of anti-inflammatory cytokines such as adiponectin. Adiponectin is secreted by white adipocytes and is well established as an insulin-sensitizing hormone that exerts control over a multitude of metabolic processes in numerous tissues. Adiponectin serum concentrations are higher in females both in humans and in rodents. ${ }^{3}$ Studies show that in obesity, total adiponectin concentrations decrease, but weight loss counteracts this decrease. ${ }^{5}$ Moreover, hypoadiponectinemia also plays a role in the development of cardiovascular disease as low serum adiponectin levels are predictors of atherosclerosis and myocardial infarction. Serum adiponectin levels have also been found to be inversely correlated to the intima thickness, a marker of carotid atherosclerosis. ${ }^{5}$ White adipose tissue can be divided into 2 major compartments - the visceral and subcutaneous adipose tissue. Increased VAT portends higher cardiometabolic risk as compared to SAT expansion. ${ }^{6}$ Obesity is also accompanied by chronic inflammation, with tissue infiltration of macrophages and other immune cells which are also able to produce proinflammatory cytokines, which in return can increase the risk of developing cardiovascular disease.

\section{${ }^{18}$ F-FDG IMAGING OF ADIPOSE TISSUE}

2-deoxy-2-18F-fluoro-D-glucose $\left({ }^{18} \mathrm{~F}-\mathrm{FDG}\right)$ is a radiolabelled glucose analogue which is clinically used to assess metabolic activity of different organs. In the past few years, ${ }^{18} \mathrm{~F}$-FDG uptake in adipose tissue was used as a surrogate marker for increased metabolic activity in all types of adipose tissue - both white and brown adipose tissue. ${ }^{7,8}$ A previously published study shows that different inflammation markers (like high sensitive CRP or the SUVmax values of the spleen and bone marrow) significantly correlate with VAT SUVmax whereas no correlations were noticed with VAT SUVmean, in part due to the fact that SUVmean of VAT is in several studies lower in obese participants as compared to lean, healthy participants. ${ }^{9}$ Therefore, VAT SUVmax or VAT TBR is most commonly used to express the adipose tissue inflammation. A study comparing metabolically healthy obese individuals and metabolically abnormal obese with metabolically healthy lean individuals showed that in all compared groups, VAT ${ }^{18}$ F-FDG uptake was higher than the SAT uptake. Interestingly, both metabolically healthy and abnormal obese individuals had similar VAT ${ }^{18}$ F-FDG uptake which was significantly lower than the VAT ${ }^{18}$ F-FDG uptake of metabolically lean individuals. ${ }^{6}$ This suggests that obese individuals, independent of their metabolic profile, may have abnormal adipose tissue glucose handling or may possess more insulin-resistant adipocytes. Moreover, this could also reflect abnormal adipose tissue perfusion, vascular function, and capillary density. ${ }^{10}$

\section{ASSESSMENT OF ARTERIAL INFLAMMATION USING ${ }^{18}$ F-FDG PET/CT}

In the past few years, the use of ${ }^{18} \mathrm{~F}$-FDG PET/CT has emerged as a reliable tool in the assessment of arterial inflammation. Thus, functional imaging of arterial inflammation helps identify patients at risk of plaque rupture and it can also characterize atherosclerotic plaques on a molecular level. It is widely accepted that ${ }^{18}$ F-FDG uptake in the vessels indicates the degree of macrophage infiltration and the levels of inflammatory gene expression in atherosclerotic plaques, making ${ }^{18} \mathrm{~F}$ FDG a very important and useful tool in modern metabolic research. Furthermore, ${ }^{18}$ F-FDG uptake in adipose tissue and vessels gives important information about the metabolic activity in a non-invasive manner. ${ }^{11}$ Based on a recent position paper, both the segmentation of the vessels and imaging protocols have been standardized. Briefly, the aorta can be divided into 4 main segments - ascending aorta, aortic arch, descending aorta, and abdominal aorta; other vessels that are usually used for plaque quantification are the carotid arteries and the iliac arteries. The PET acquisition is started $2 \mathrm{~h}$ after the injection of the radiotracer. The quantification of ${ }^{18}$ F-FDG uptake in atherosclerotic plaques uses TBR instead of SUV values. TBR is calculated as the ratio of SUVmax of the arterial segment divided by SUVmean of the V. jugularis for the carotid arteries, V. cava superior for the ascending aorta and aortic arch, and V. cava inferior for the descending aorta, abdominal aorta, and iliac arteries. TBR is used instead of SUV as the use of a ratio between two measurements limits the effects on signal quantification of errors in patient weight and in the injected tracer dose and imaging time point. ${ }^{12}$

In their paper, Toutouzas and colleagues conducted a well-designed observational, case-controlled study including 20 patients with heFH, 20 patients with $\mathrm{FCH}$, as well as 20 healthy controls. In their work, the authors show that FCH patients had significantly lower VAT metabolic activity as compared to patients with heFH or controls. These patients also showed a significantly higher VAT volume as compared to heFH patients and controls. Interestingly, FCH patients were mostly obese males as compared to heFH patients or controls which had a 50:50 sex distribution and were 
lean. The fact that obese individuals have lower ${ }^{18} \mathrm{~F}$ FDG uptake in VAT has been previously shown, ${ }^{6}$ but it is intriguing that $\mathrm{FCH}$ patients do not show marked adipose tissue inflammation, i.e., increased VAT metabolic activity. No differences were observed in SAT metabolic activity or SAT volume between $\mathrm{FCH}$ patients and heFH patients, whereas a marked difference was observed between FCH patients and controls, an effect which can be partly explained by the patients' weight. Moreover, no differences were noticed in C-reactive protein levels between the study cohorts, as compared to a previous study published by Toutouzas and colleagues where there were significant differences in the inflammation parameters between the two groups. ${ }^{13}$ Notably, $\mathrm{FCH}$ patients had significant lower adiponectin levels indicating a higher inflammatory state as compared to heFH patients and controls. However, most patients $(95 \%)$ in the $\mathrm{FCH}$ group were male and it is widely accepted that circulating adiponectin levels are lower in males. ${ }^{3}$ Moreover, FCH patients were obese as compared to heFH patients and controls, a state which is associated with hypoadiponectinemia and generally with increased cardiovascular risk.

Another interesting finding of this study was that the VAT volume was the strongest negative predictor of serum adiponectin levels, an effect partly mediated through VATs metabolic activity. The authors further performed unsupervised hierarchical clustering of FCH and heFH patients based on VAT metabolic activity, VAT volumetrics and circulating adiponectin levels. Based on the adiponectin levels, two separate clusters (cluster A - low adiponectin and cluster B - high adiponectin) were defined. Cluster A subsequently presented two lower clusters: a cluster with low VAT metabolic activity and high VAT volume and a cluster with high VAT metabolic activity and variable VAT volume. Cluster A consisted of both FCH and heFH patients. A limitation of this very interesting study is the lack of comparison between the two subclusters within cluster A. A direct comparison between the two subclusters could have given more insight in the metabolic phenotype (like BMI, WHR, or serum lipids) of these individuals. Cluster B, the high adiponectin group, mainly showed low VAT volumes and high VAT metabolic activity, yet this group mostly consisted of lean individuals, where high VAT ${ }^{18}$ F-FDG uptake was previously described. ${ }^{6}$ Furthermore, the levels of circulating adiponectin also correlated with the vascular inflammation of the abdominal aorta as shown by mean TBRmax. The two main clusters also had significant different aortic TBRs, with the hypoadiponectinemic cluster presenting significant higher aortic TBR values as compared to cluster B. A further limitation of this study was the fact that the authors only included the abdominal aorta. It would have been interesting to see correlations between TBR values of the remaining aortic segments.

The authors conclude that VAT phenotype could account for the differences in arterial inflammation levels between FCH and heFH. As previously shown in obese individuals, excess visceral adiposity or high VAT metabolic activity could lead to a decrease in serum adiponectin levels promoting thus vascular inflammation in FCH patients. The fact that the cluster including hypoadiponectinemic individuals included both $\mathrm{FCH}$ and heFH patients and both patients with high and low VAT metabolic activity leaves room for new research opportunities. It has been already suggested that $\mathrm{FCH}$ patients have lower adiponectin levels, ${ }^{14}$ but adiponectin levels in heFH patients are still weakly defined. Furthermore, it would be interesting to understand if there are differences in the adipose tissue phenotype and arterial inflammation in heFH patients with different adiponectin levels and a lean phenotype.

The study of Toutouzas et al found that in familial dyslipidemias the VAT phenotype is associated with the degree of arterial inflammation, an effect which is in part mediated by adiponectin. The authors also state that hypoadiponectinemia in the presence of either excess visceral adiposity or high VAT metabolic activity is associated with increased levels of arterial inflammation in FCH patients. This statement is controversial due to lacking analyses of the hypoadiponectinemic cluster.

The study of Toutouzas et al provides new insights into the mechanisms of vascular disease in familial dyslipidemia subtypes and the role of adipose tissue in this matter but also opens new exciting research opportunities on hypoadiponectinemia in familial dyslipidemia.

\section{References}

1. Bouhairie VE, Goldberg AC. Familial hypercholesterolemia. Endocrinol Metab Clin N Am 2016;45(1):1-16.

2. Taghizadeh E, Esfehani RJ, Sahebkar A, Parizadeh SM, Rostami D, Mirinezhad M, et al. Familial combined hyperlipidemia: An overview of the underlying molecular mechanisms and therapeutic strategies. IUBMB Life 2019;71(9):1221-9.

3. Burhans MS, Hagman DK, Kuzma JN, Schmidt KA, Kratz M. Contribution of Adipose Tissue Inflammation to the Development of Type 2 Diabetes Mellitus. Compr Physiol 2018;9(1):1-58.

4. Longo, M., Zatterale, F., Naderi, J., Parrillo, L., Formisano, P., Raciti, G.A., et al., 2019. Adipose Tissue Dysfunction as Determinant of Obesity-Associated Metabolic Complications. Int J Mol Sci 20(9).

5. Nigro E, Scudiero O, Monaco ML, Palmieri A, Mazzarella G, Costagliola $\mathrm{C}$, et al. New insight into adiponectin role in obesity and obesity-related diseases. Biomed Res Int. 2014;2014:658913.

6. Oliveira AL, Azevedo DC, Bredella MA, Stanley TL, Torriani M. Visceral and subcutaneous adipose tissue FDG uptake by PET/CT 
in metabolically healthy obese subjects. Obesity (Silver Spring) 2015;23(2):286-9.

7. Christen T, Sheikine Y, Rocha VZ, Hurwitz S, Goldfine AB, Di Carli $\mathrm{M}$, et al. Increased glucose uptake in visceral versus subcutaneous adipose tissue revealed by PET imaging. JACC Cardiovasc Imaging 2010;3(8):843-51

8. Kulterer, O.C., Niederstaetter, L., Herz, C.T., Haug, A.R., Bileck, A., Pils, D., et al., 2020. The presence of active brown adipose tissue determines cold-induced energy expenditure and oxylipin profiles in humans. J Clin Endocrinol Metab 105(7).

9. Pahk K, Kim EJ, Lee YJ, Kim S, Seo HS. Characterization of glucose uptake metabolism in visceral fat by 18 F-FDG PET/CT reflects inflammatory status in metabolic syndrome. PLoS ONE. 2020;15(2):e0228602.

10. Farb MG, Ganley-Leal L, Mott M, Liang Y, Ercan B, Widlansky $\mathrm{ME}$, et al. Arteriolar function in visceral adipose tissue is impaired in human obesity. Arterioscler Thromb Vasc Biol 2012;32(2):46773.

11. Bucerius J, Mani V, Wong S, Moncrieff C, Izquierdo-Garcia D, Machac J, et al. Arterial and fat tissue inflammation are highly correlated: a prospective 18F-FDG PET/CT study. Eur J Nucl Med Mol Imaging 2014;41(5):934-45.

12. Bucerius J, Hyafil F, Verberne HJ, Slart RH, Lindner O, Sciagra R, et al. Position paper of the Cardiovascular Committee of the European Association of Nuclear Medicine (EANM) on PET imaging of atherosclerosis. Eur $\mathrm{J}$ Nucl Med Mol Imaging 2016;43(4):780-92.

13. Toutouzas K, Skoumas J, Koutagiar I, Benetos G, Pianou N, Georgakopoulos A, et al. Vascular inflammation and metabolic activity in hematopoietic organs and liver in familial combined hyperlipidemia and heterozygous familial hypercholesterolemia. J Clin Lipidol 2018;12(1):33-43.

14. van der Vleuten GM, van Tits LJ, den Heijer M, Lemmers H, Stalenhoef AF, de Graaf J. Decreased adiponectin levels in familial combined hyperlipidemia patients contribute to the atherogenic lipid profile. J Lipid Res 2005;46(11):2398-404.

Publisher's Note Springer Nature remains neutral with regard to jurisdictional claims in published maps and institutional affiliations. 